\title{
Programmed cell death ligand-1 expression in tumor and immune cells is associated with better patient outcome and decreased tumor-infiltrating lymphocytes in uveal melanoma
}

\author{
Pablo Zoroquiain ${ }^{1,2,3} \cdot$ Evangelina Esposito $^{1} \cdot$ Patrick Logan $^{1} \cdot$ Sultan Aldrees $^{1} \cdot$ Ana Beatriz Dias $^{1}$ • \\ Jose Joao Mansure ${ }^{1}$ - Daniela Santapau ${ }^{1} \cdot$ Ciro Garcia $^{1} \cdot$ Maria Antonia Saornil $^{1} \cdot$ Rubens Belfort Neto $^{1,3}$. \\ Miguel N. Burnier ${ }^{1,3}$
}

Received: 2 October 2017 / Revised: 1 February 2018 / Accepted: 2 February 2018 / Published online: 26 March 2018

(c) United States \& Canadian Academy of Pathology 2018

\begin{abstract}
Programmed cell death-1/ligand (PD-1/PD-L1) interaction negatively regulates T cell activity. PD-L1 expression in tumor cells, antigen-presenting cells, and lymphocytes of the tumor microenvironment is associated with response to treatment with PD-1/PD-L1 inhibitors, but there is still debate on the cutoff value that correlates with responders. In uveal melanoma (UM), $40 \%$ of patients will develop liver metastases and, amongst them, $90 \%$ will succumb to their disease. The aim of this study was to analyze PD-L1 expression as a prognostic marker and as a possible therapeutic target for UM. Sixty-seven enucleated eyes from UM patients with relevant clinical information were analyzed. Univariate and multivariate analysis were used to evaluate association of PD-L1 with survival. PD-L1 expression was positive relatively to tumor cells, immune cells, and the tumor and tumor-infiltrating immune cell group scoring in 46, 34 and 55\% of the cases, respectively. On univariate analysis, tumor cells and the tumor and tumor-infiltrating immune cell group PD-L1 expression was associated with a longer metastasis-free survival $(P=0.04$ and $P=0.007)$. However, on multivariate analysis, only the tumor and tumor-infiltrating immune cell group positivity was associated with longer metastasis-free survival $(P=0.01)$. Furthermore, tumor cells and the tumor and tumor-infiltrating immune cell group PD-L1 expression was associated with decreased tumor-infiltrating lymphocytes $(P=0.02)$. PD-L1, when expressed in uveal melanoma, is associated with better patient outcome and decreased tumor-infiltrating lymphocytes. These results support the consideration of anti-PD-1/PD-L1 therapy in uveal melanoma. To determine the best cutoff value, further studies from patients enrolled in clinical trials treated with PD-1/PDL1 inhibitors are necessary.
\end{abstract}

The present study was presented as an oral presentation in the United States and Canada Academy of Pathology (USCAP) on March 2017 and as a poster presentation in the Association for Research in Visual science and Ophthalmology (ARVO) on May 2017.

Electronic supplementary material The online version of this article (https://doi.org/10.1038/s41379-018-0043-5) contains supplementary material, which is available to authorized users.

Pablo Zoroquiain

zoroquiain@gmail.com

1 McGill Ocular Pathology Laboratory McGill University Health Centre Montreal QC Canada

2 Pathology Department, School of Medicine, Pontificia Universidad Católica de Chile, Santiago, Chile

3 Ophthalmology Department, School of Medicine, Universidade Federal de São Paulo, São Paulo, Brazil

\section{Introduction}

Uveal melanoma is the most common primary intraocular malignancy in adults [1]. Despite better understanding of the disease and new treatment modalities, $40 \%$ of patients will develop liver metastasis within 5 years. Furthermore, patients can develop metastasis even up to 20 years after initial diagnosis $[2,3]$.

For many years, enucleation has been the standard treatment for uveal melanoma. Clinicians and scientists believed that this modality would lower the likelihood of metastasis. However, it is now well known that there is no difference in clinical outcome regardless of primary tumor treatment [3]. Despite all of the technical advances in treating the primary tumor, including radiotherapy and chemotherapy, the incidence of metastasis has remained relatively unchanged over the past four decades [4]. 
Several hypotheses have been proposed to address this conundrum. A widely accepted theory stipulates that, unlike cutaneous melanomas that usually have well-documented growth history in visible areas, uveal melanomas are usually quite large before the appearance of symptoms leading the patient to seek medical attention. In support of this hypothesis, it has been shown that most patients have detectable circulating malignant cells at the time of the diagnosis, regardless of the treatment modality $[5,6]$. Moreover, studies in mice have revealed that metastatic cells are capable of remaining dormant in the liver with little or no cellular division for up to 7 weeks [7]. Thus, it is conceivable that cells leave the eye in the early stages of development, before the tumors are clinically detected. Consequently, the metastatic seeds are already present, rendering even the most aggressive primary tumor treatments irrelevant with respect to metastatic disease.

Current metastatic anti-cancer treatments are focused on the longstanding concept of manipulating the immune system to fight cancer. These ideas have been invigorated by the discovery and development of effective blockers of immune pathway checkpoints, such as ipilumab (antiCTLA-4), the first Food and Drug Administration (FDA)approved immunomodulatory drug [8]. Another checkpoint of interest in the immune pathway is the programmed cell death-1/programmed cell death ligand-1 (PD-1/PD-L1). The PD-1/PD-L1 pathway is a co-inhibitory pathway that negatively regulates $\mathrm{T}$ cell activity [9]. PD-1 is a receptor that is expressed on $\mathrm{T}$ lymphocytes and has two ligands: PD-L1 (also known as B7-H1 or CD274) and PD-L2 belonging to the $\mathrm{B} 7$ superfamily. Both are expressed on antigen-presenting cells, other regulatory and helper $\mathrm{T}$ lymphocytes, and neoplastic cells [9]. PD-L1 is also present in other tissues and is positively regulated by interferon- $\gamma$. PD-L1 can also bind to CD80-expressing T lymphocytes [10], whereas PD-L2 can bind to the repulsive guidance molecule B.

In neoplasia, the importance of the PD-1/PD-L1 axis lies in the ability of tumor cells to create $\mathrm{T}$ cell depletion [11], giving them an adaptive resistance to the immune system. Interferon $-\gamma$ activates adaptive immunity and also leads to a greater expression of PD-L1, resulting in the neutralization of the adaptive immune system [12].

Anti-PD-1/PD-L1 therapy has been one of the greatest achievements in modern oncology. To date, two anti-PD-1 drugs have been FDA approved: pembrolizumab and nivolumab. These drugs demonstrated an objective response in $40 \%$ of the patients with metastatic cutaneous melanoma $[11,13,14]$.

PD-L1 expression studies have generated a great deal of interest with respect to the searching of a marker for antiPD-L1 treatment response. Clinical studies with anti-PD-1/ PD-L1 axis drugs have shown an association between patients responding to therapy and expressing PD-L1 on the surface of tumor cells. Early studies showed that an objective response was achieved in $36 \%$ of patients whose tumors expressed PD-L1 in more than $5 \%$ of cells. On the other hand, those who had $<5 \%$ of tumor expression had no response to treatment [15]. Subsequent studies have used different cutoff values ranging from $1 \%$ and up to $50 \%$. However, there is a lack of consensus on a universal cutoff point [16]. Furthermore, it is important to recognize that different studies have used different antibodies and detection methods, which adds to the difficulty in interpreting data [17].

In uveal melanoma, studies have shown conflicting results. In vitro studies by flow cytometry and mRNA expression by reverse transcriptase-polymerase chain reaction suggest constitutive expression of PD-L1 in five of nine cell lines from primary tumors and in one of five metastatic cell lines. However, for all primary and metastatic PD-L1negative cell lines, the expression was stimulated when cells were exposed to interferon- $\gamma$. It was further demonstrated that tumor-lymphocyte interaction was also modified by PD-L1. When uveal melanoma cells were exposed to interferon-gamma, the secretion of IL-2 by the co-cultured lymphocytes diminished. Similarly, if PD-L1 was blocked in the same experimental setup, IL-2 levels increased [18]. In this same study, PD-L1 expression was evaluated by immunohistochemistry in five enucleated eyes of uveal melanoma patients and negative expression was found in all cases. However, other study using another monoclonal antibody and flow cytometry demonstrated that the OCM-1, 92.1 and OMM-1 cell lines constitutively expressed low levels of PD-L1 [19], which was not evidenced in the initial studies of Yang et al. [18].

These discrepancies of PD-L1 expression in uveal melanoma are no exception. It was also observed in other neoplasms that the immunohistochemical evaluation of PD-L1 may have inconsistent results and nonreproducibility due to the lack of specificity of the available antibodies at that time, such as the MIH1 clone [20]. Numerous studies of malignancies from different origins have shown conflicting results [11,21]. Fortunately, a new generation of commercially available antibodies has been produced and has contributed to more standardized results [11, 22].

Due to the importance of finding new treatment targets for systemic uveal melanoma disease, we attempt to validate the specificity of a commercially available anti-PD-L1 antibody and to analyze PD-L1 expression in uveal melanoma as a potential target for therapy. Also, we correlate PD-L1 expression with histologic prognostic factors and clinical outcome. Understanding the role of PD-L1 in UM may lead to a novel directed therapy approach using immune therapies targeting the PD-1/PD-L1 pathway. 


\section{Materials and methods}

\section{Validation step}

In order to validate our immunohistochemical procedure, knockdown experiments using the OCM-1 uveal melanoma cell line were performed and placenta was used as a positive control to detect specific staining in the syncytiotrophoblast. Moreover, Western blot analysis was performed to visualize the protein in the same cell line (Supplemental file 1)

\section{Uveal melanoma cases}

After validation of the antibody, opto-pupilary sections of uveal melanoma enucleation specimens were retrieved from the Henry C Witelson Ocular Pathology Laboratory archives. The following clinical information was recorded: age, gender, location, date of enucleation, history of prior radiotherapy, and follow-up in months. Patients were categorized as having metastatic disease if they had on record a biopsy of the lesion or if they had images consistent with the diagnosis.

For morphology, cell type was classified according to the modified Callender's classification [23]. The presence of mitotic figures was scored as low or high (cutoff value: 10 mitosis in 40 consecutive high power fields) [24]. Ciliary body involvement and presence of closed vascular loops on hematoxylin-eosin was also assessed [25]. The largest tumor dimension was measured in $\mathrm{mm}$ by B-scan ultrasound before enucleation and was considered low if less than $15 \mathrm{~mm}$ and high if $\geq 15 \mathrm{~mm}$. Follow-up was measured in months. The TNM staging was assessed according to the AJCC 7th edition.

Tumor-infiltrating lymphocyte and tumor-associated macrophage analysis was conducted by two ocular pathologists blinded to PD-L1 expression and clinical parameters. The threshold for positive tumor-infiltrating lymphocytes that was set was more than 100 lymphocytes in 20 high power fields as described previously [26], while the presence of tumor-associated macrophages was determined semi-quantitatively as low or high as previously described [27].

Data acquisition was in accordance with Canadian and Quebec provincial legislation and the tenets of the Declaration of Helsinki.

\section{Immunohistochemistry}

Automated immunohistochemistry was performed using the Ventana benchmark machine according to the manufacturer's recommended protocol (Ventana Medical Systems, Inc., Tucson, AZ, USA). The fully automated process involved barcode labeling the slides, baking the slides, solvent-free deparaffinization, and CC1-based antigen retrieval (Tris-EDTA buffer $(\mathrm{pH}$ 8.0)). Slides were incubated with a monoclonal anti-human PD-L1 antibody (Cell Signaling, E1L3N clone) at a dilution of 1:50 for $30 \mathrm{~min}$ at $37^{\circ} \mathrm{C}$ followed by application of a biotinylated secondary antibody for $8 \mathrm{~min}$ at $37^{\circ} \mathrm{C}$ and then an avidin/streptavidin enzyme conjugate complex was added. Finally, Fast Red was used as the chromogenic substrate and slides were counterstained with hematoxylin. On each automated immunohistochemistry cycle, a placenta section was added as an external internal control. Inner control was determined by cytoplasmic positivity for retinal pigmented epithelium cells as described previously [28].

\section{Immunohistochemical evaluation}

The slides were independently evaluated by two ocular pathologists using whole slide images obtained with a digital slide scanner (Aperio ScanScope AT Turbo, Leica microsystem, Concord, ON, Canada) and analyzed with Aperio Image Analyzer. As indicated earlier for phase I clinical trials in other solid tumors, PD-L1 positivity was defined by a threshold of $5 \%$ of tumor cell expression. To be considered positive, the cells must have a strong cytoplasmic expression with membrane-accentuating or single membrane pattern.

Recent studies have shown that tumor microenvironment has a great impact on the biological behavior of the neoplasia and can modify immune response [29]. Moreover, it has been described that PD-L1 expression in antigenpresenting cells and lymphocytes exerts a negative regulation of the immune system [30]. The POPLAR study group has also addressed this issue in the past and stated that tumor response was associated with positivity in either the tumor cells or immune cells [31]. Therefore, we chose to adopt an improved cutoff value where tumor cells and immune cells are analyzed based on morphology, as described by Fehrenbacher et al. [31]. In this system, PD-L1 positivity is defined by a $5 \%$ threshold of immune cells and the tumor or immune cell group, similarly to what has been performed [32].

\section{Statistical analysis}

To determine if the predictive factors described previously are applicable to the population and to evaluate the prognostic capacity of PD-L1 in the study group, Kaplan-Meier survival curves were traced and the differences were estimated with the log-rank test. $\chi^{2}$ was used to analyze the association between PD-L1 expression and clinical-pathological data, including extraocular extension, tumor-infiltrating lymphocytes, tumor-associated 
macrophages, cell type, largest tumor dimension, pTNM status, gender, mitotic figures, and age. Differences were considered significant at $P<0.05$. Subsequently, prognostic factors were evaluated using multivariate analysis using the Cox regression method. The statistics were performed using Microsoft Excel, GraphPad Prism 5.0 software (Microsoft Corp., Redmond, WA, USA; GraphPad Software Inc., La Jolla, CA, USA) and SPSS software v9.0 (IBM, Armonk, NY, USA).

\section{Results}

\section{Analysis of patients with uveal melanoma}

Seventy-seven cases of uveal melanoma from 77 patients were analyzed. Initial reviews assessing positive control was performed and nine cases were found to have a negative internal control and were therefore were excluded from the subsequent analysis. In addition, one case represented a tumorectomy and did not have a valid internal control so it was also excluded.

Finally, 67 cases had positive internal controls and were analyzed in detail. In this cohort, the male:female ratio was 1.8:1 and the mean age was $63.46 \pm 14.24$ years. The mean largest tumor dimension was $14.98 \pm 4.3 \mathrm{~mm}$. Sixty-five percent of uveal melanoma cases occurred in the left eye. None of the cases had prior history of radiation. Clinicopathological characteristics are described in Table 1.

\section{Metastatic group}

Twenty-five of the 67 patients (37\%) had metastatic disease. In the metastatis group, the mean largest tumor dimension was $16.2 \mathrm{~mm}$ (median 20, range 9.6-24.6 mm). Eighty-four percent of the cases were of mixed cell type, $72 \%$ of cases had tumor-associated macrophages, $40 \%$ had tumorinfiltrating lymphocytes, $24 \%$ had extraocular extension, and $52 \%$ of cases had ciliary body involvement. The follow-up period in this group had a median of 24 months (range 6-95). All cases had hepatic metastases.

\section{Group without metastases}

Forty-two of 67 patients $(63 \%)$ had no metastatic disease. The follow-up period in this group ranges from 2 to 248 months (median 58). In this particular group, the mean largest tumor dimension was $14.3 \mathrm{~mm}$ (median 14, range $8-18.5 \mathrm{~mm}$ ). Sixty percent of the cases were of mixed cell type, $41 \%$ of cases had tumor-associated macrophages, $29 \%$ had tumor-infiltrating lymphocytes, $21 \%$ had extraocular extension, and $31 \%$ of cases had ciliary body involvement.
An univariate log-rank analysis of the Kaplan-Meier curves for each prognostic factor showed significantly lower metastasis-free survival for the age group $>65$ years $(P=$ $0.03)$, the mixed cell type $(p=0.005)$, the presence of tumor-associated macrophages $(p=0.045)$, the presence of tumor-infiltrating lymphocytes $(p=0.02)$, and ciliary body involvement $(P=0.03)$. In contrast, mitotic index, vascular loops, largest tumor dimension, and T stage of the TNM classification had no significant prognostic influence in our series $(P>0.05)$.

\section{Expression of PD-L1}

The expression of PD-L1 was observed in the cytoplasm of cells with an accentuation in the cellular membranes; the distribution was either patchy or diffuse (Figs. 1 and 2, respectively). In all negative cases, PD-L1 expression was observed in the retinal pigmented epithelium confirming the antigenicity of the sample.

\section{Tumor PD-L1 expression scoring system (conventional grading)}

Following the conventional grading, where a positive score corresponds to PD-L1 expression in more than $5 \%$ of tumor cells, for $46 \%$ of the cases were PD-L1 positive. PD-L1 positivity was observed in $50 \%$ of the cases in the group without metastatic disease, whereas PD-L1 was positive in $40 \%$ of the cases with metastatic disease.

An univariate analysis showed an association of PD-L1 expression to a decreased number of tumor-infiltrating lymphocytes $(P=0.04)$. The result of the univariate analysis is summarized in Table 1.

Log-rank analysis of the Kaplan-Meier curves showed that PD-L1 expression is associated with metastasis-free survival $(P=0.038$, Fig. 3a). Since PD-L1 showed a correlation with metastasis-free survival, it was decided to analyze whether this correlation is independent of other prognostic factors. It was observed that neoplastic PD-L1 expression in this cohort was not an independent prognostic factor. The summary of the regression analysis is shown in Table 2 .

\section{Infiltrating immune cell PD-L1 expression scoring system}

To assess microenvironment, the expression of PD-L1 was analyzed in infiltrating immune cells, independently of tumor cells. It was observed that $34 \%$ of the cases were positive for PD-L1.

As demonstrated in Table 1, univariate analysis showed an association between immune cell PD-L1 and a lower age at diagnosis $(P=0.009)$. Moreover, immune cell PD-L1 
Table 1 Clinicopathologic characteristics and univariate analysis of the likelihood of tumoral PD-L1 (TC), tumor-infiltrating immune cells (IC), or the tumor and tumor-infiltrating immune cell group (TC/IC) associated with other prognostic factors in the study population

\begin{tabular}{|c|c|c|c|c|c|c|c|c|c|c|}
\hline & \multirow[t]{2}{*}{$N(\%)$} & \multicolumn{2}{|c|}{ PD-L1 tumor cells } & \multirow[t]{2}{*}{$P$ value } & \multicolumn{2}{|c|}{ PD-L1 immune cells } & \multirow[t]{2}{*}{$P$ value } & \multicolumn{2}{|c|}{$\begin{array}{l}\text { PD-L1 tumor and } \\
\text { immune cells }\end{array}$} & \multirow[t]{2}{*}{$P$ value } \\
\hline & & Negative & Positive & & Negative & Positive & & Negative & Positive & \\
\hline \multicolumn{11}{|l|}{ Age (years) } \\
\hline$\geq 65$ & $36(54)$ & 15 & 16 & 0.47 & 29 & 7 & $0.009 *$ & 11 & 20 & 0.14 \\
\hline$<65$ & $31(46)$ & 21 & 15 & & 15 & 16 & & 20 & 16 & \\
\hline \multicolumn{11}{|l|}{ Gender } \\
\hline Male & $43(69)$ & 21 & 22 & 0.31 & 26 & 17 & 0.29 & 16 & 27 & 0.07 \\
\hline Female & $24(31)$ & 15 & 9 & & 17 & 6 & & 15 & 9 & \\
\hline \multicolumn{11}{|l|}{ Cell type } \\
\hline Spindle & $21(31)$ & 8 & 13 & 0.11 & 11 & 10 & 0.17 & 6 & 15 & 0.07 \\
\hline Mixed & $46(69)$ & 28 & 18 & & 33 & 13 & & 25 & 21 & \\
\hline \multicolumn{11}{|l|}{ TAM } \\
\hline Absent & $32(48)$ & 20 & 12 & 0.22 & 23 & 9 & 0.44 & 17 & 15 & 0.33 \\
\hline Present & $35(52)$ & 16 & 19 & & 21 & 14 & & 14 & 21 & \\
\hline \multicolumn{11}{|l|}{ Vascular loops } \\
\hline Absent & $32(48)$ & 19 & 21 & 0.32 & 23 & 17 & 0.07 & 15 & 25 & 0.08 \\
\hline Present & $35(52)$ & 16 & 10 & & 21 & 5 & & 16 & 10 & \\
\hline \multicolumn{11}{|l|}{ TIL } \\
\hline Absent & $45(67)$ & 20 & 25 & $0.04 *$ & 27 & 17 & 0.18 & 16 & 29 & $0.02 *$ \\
\hline Present & $22(33)$ & 16 & 6 & & 18 & 5 & & 15 & 7 & \\
\hline \multicolumn{11}{|l|}{$\mathrm{CB}$ infiltration } \\
\hline No infiltration & $42(63)$ & 19 & 22 & 0.14 & 25 & 16 & 0.43 & 16 & 25 & 0.21 \\
\hline Infiltrated & $28(37)$ & 17 & 9 & & 19 & 7 & & 17 & 9 & \\
\hline Mitotic figures & & & & 1 & & & & & & \\
\hline$<10$ & $60(89)$ & 32 & 28 & & 37 & 23 & 0.09 & 27 & 33 & 0.70 \\
\hline 10 or more & $7(11)$ & 4 & 3 & & 7 & 0 & & 4 & 3 & \\
\hline \multicolumn{11}{|l|}{ LTD } \\
\hline$\geq 15 \mathrm{~mm}$ & $32(48)$ & 14 & 18 & 0.15 & 26 & 9 & 0.13 & 12 & 20 & 0.23 \\
\hline$<15 \mathrm{~mm}$ & $35(52)$ & 22 & 13 & & 18 & 14 & & 19 & 16 & \\
\hline \multicolumn{11}{|l|}{ pTNM } \\
\hline $\mathrm{T} 1-\mathrm{T} 2$ & $21(31)$ & 9 & 12 & 0.29 & 11 & 10 & 0.17 & 7 & 14 & 0.19 \\
\hline $\mathrm{T} 3-\mathrm{T} 4$ & $46(69)$ & 27 & 19 & & 33 & 13 & & 24 & 22 & \\
\hline
\end{tabular}

TAM tumor-associated macrophages, $T I L$ tumor-infiltrating lymphocytes, $P D$ - $L 1$ programmed cell death-1/ligand, LTD larger tumor dimension, $C B$ ciliary body, TNM tumor, node, metastasis staging

expression did not have any significant association with prognosis $(P=0.33$, Fig. 3b).

\section{Tumor and tumor-infiltrating immune cell PD-L1 expression scoring system}

The expression of PD-L1 was analyzed considering PD-L1 expression in both tumor and tumor-infiltrating immune cells. With this new cutoff value, it was observed that $54 \%$ of the cases were positive for PD-L1. Moreover, $60 \%$ of patients without metastases were positive for PD-L1, compared to $44 \%$ of patients who had metastases.
A univariate analysis showed that the expression of PDL1 in the tumor and tumor-infiltrating immune cell group is associated with a decrease in tumor-infiltrating lymphocytes $(P=0.02)$. The evaluation is summarized in Table 1 .

Log-rank analysis of the Kaplan-Meier survival curves for this new threshold value shows that PD-L1 expression in more than $5 \%$ of the tumor and tumor-infiltrating immune cell group was associated with a longer metastasis-free survival $(P=0.007$, Fig. $3 c)$.

As with the standard cutoff value, the tumor and tumorinfiltrating immune cell group PD-L1 expression showed a correlation with metastatic-free survival, a multivariate 
Fig. 1 Patchy distribution of positive PD-L1 expression in a primary uveal melanoma case: at low magnification, a large tumor is seen. On medium magnification (lower inset), patchy distribution of PD-L1 expression is observed. The membranous staining is confirmed on high power magnification (small arrowheads). On the right inset, the cytoplasmic positivity of retinal pigmented epithelium cells is seen (large arrowheads). Top left $0.4 \mathrm{x}$, top right $40 \mathrm{x}$, lower left 4,6x, lower right $40 x$
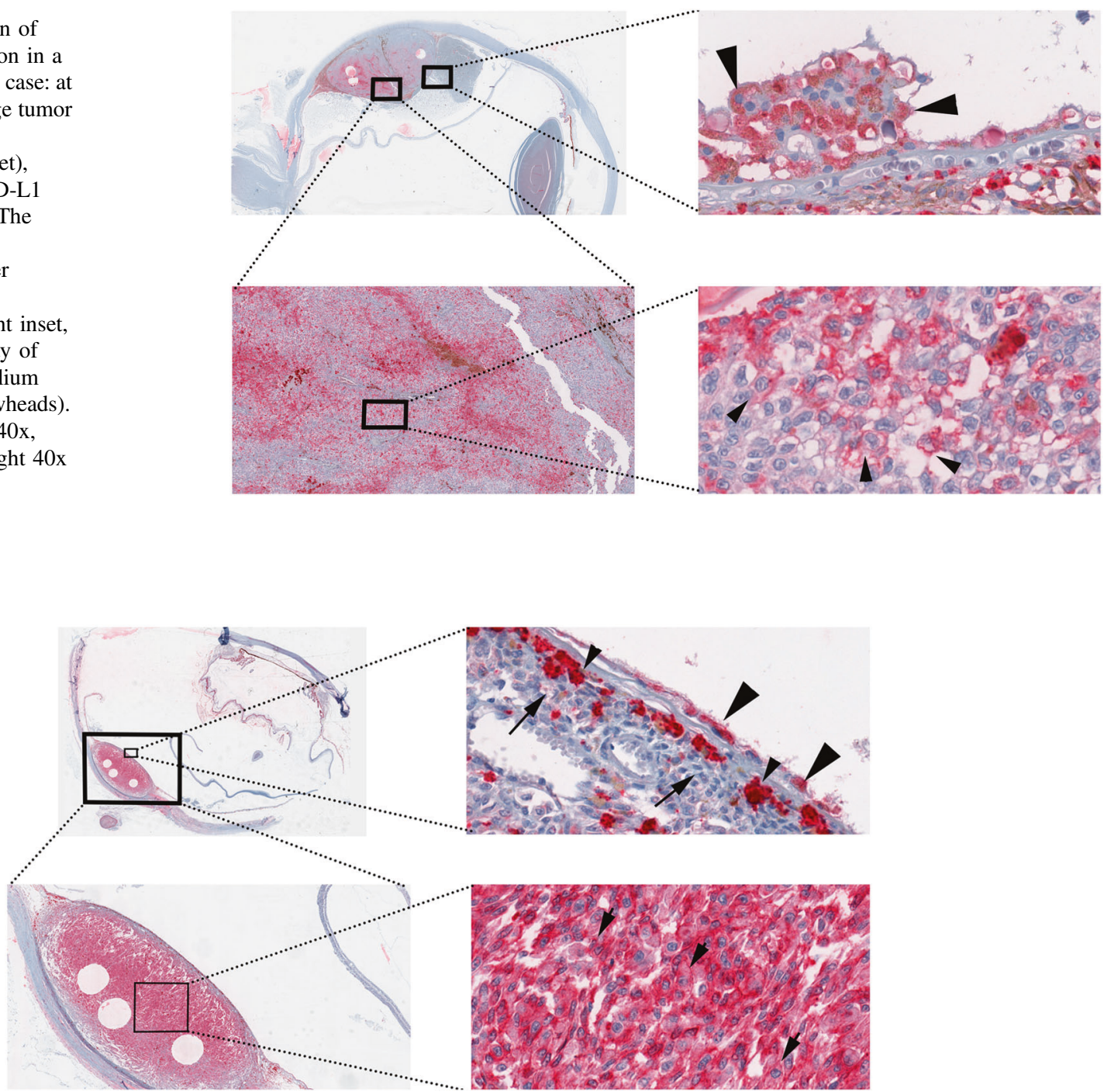

Fig. 2 Diffuse distribution of PD-L1-positive cells in a primary uveal melanoma case: at low magnification, a small flat tumor is seen. On medium magnification (lower inset), a diffuse distribution of PD-L1 expression is seen. Note that a rim of negative tumor cells is seen in the periphery. The membranous staining is confirmed on high power magnification (small arrowheads). On the right inset, the cytoplasmic positivity of retinal pigmented epithelium cells is seen (large arrowheads) and in tumor-infiltrating macrophages (small arrows). Also, note that epithelioid cells in the periphery are negative for PD-L1. Top left $0.3 \mathrm{x}$, top right $20 \mathrm{x}$, lower left $1,4 \mathrm{x}$, lower right $40 \mathrm{x}$
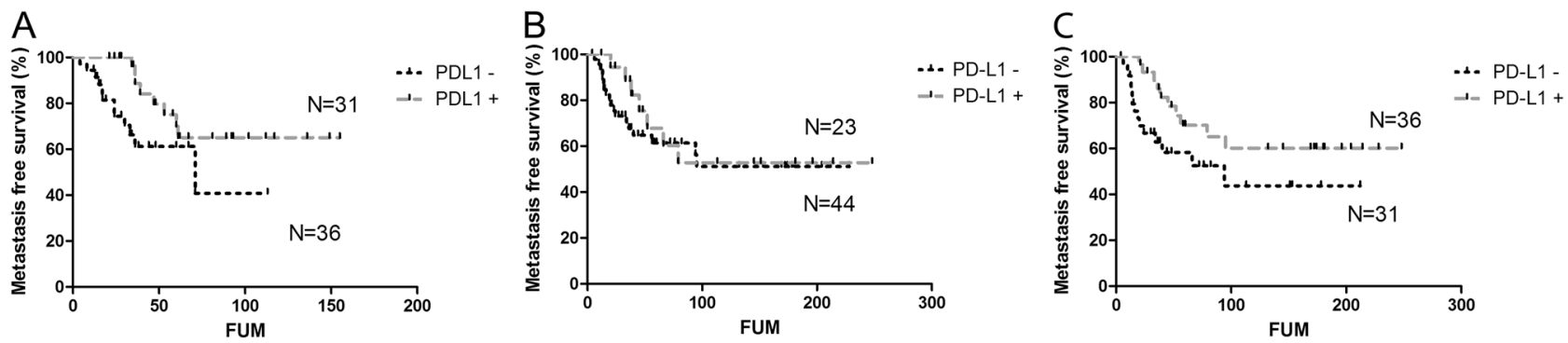

Fig. 3 Kaplan-Meier curves of metastasis-free survival (MFS) by each PD-L1 expression score analyzed in the present study. a Tumor cell PD-L1 expression. b Immune cell PD-L1 expression and $\mathbf{c}$ the tumor and tumor-infiltrating immune cell group PD-L1 expression 
Table 2 Cox's regression survival analysis of the prognostic factors including neoplastic PD-L1 expression in this cohort of uveal melanoma patients

\begin{tabular}{lllll}
\hline & $P$ value & HR & \multicolumn{2}{l}{$95.0 \%$ CI } \\
\cline { 4 - 5 } & & & Lower & Upper \\
\hline TC PD-L1 & 0.086 & 2.200 & 0.894 & 5.415 \\
TIL present & 0.683 & 1.199 & 0.503 & 2.860 \\
TAM present & 0.093 & 2.240 & 0.875 & 5.733 \\
Mixed cell type & 0.056 & 3.192 & 0.970 & 10.502 \\
CB invasion & 0.660 & 1.216 & 0.509 & 2.907 \\
Age $>$ 65 years & 0.150 & 1.022 & 0.992 & 1.054 \\
\hline
\end{tabular}

TCtumor cells, $C I$ confidence interval, $H R$ hazard ratio, $P D-L 1$ programmed cell death-1/ligand, TAM tumor-associated macrophages, $T I L$ tumor-infiltrating lymphocytes

Table 3 Cox's regression survival analysis of the prognostic factors including the tumor and tumor-infiltrating immune cell group PD-L1 expression in this cohort of uveal melanoma patients

\begin{tabular}{lllll}
\hline & $P$ value & HR & \multicolumn{2}{l}{$95.0 \%$ CI } \\
\cline { 4 - 5 } & & & Lower & Upper \\
\hline TC/IC PD-L1 & 0.036 & 0.357 & 0.137 & 0.935 \\
TIL present & 0.823 & 1.106 & 0.458 & 2.670 \\
TAM preset & 0.069 & 2.511 & 0.932 & 6.764 \\
Mixed cell type & 0.079 & 2.948 & 0.883 & 9.846 \\
CB invasion & 0.669 & 1.207 & 0.510 & 2.856 \\
Age $>$ 65 years & 0.393 & 1.014 & 0.982 & 1.047 \\
\hline
\end{tabular}

TC/IC tumor and tumor-infiltrating immune cell group, $P D-L 1$ programmed cell death-1/ligand, $C I$ confidence interval, $H R$ hazard ratio, $C B$ ciliary bodyTAM tumor-associated macrophages, TIL tumorinfiltrating lymphocytes

analysis was therefore performed. It was observed that the $>5 \%$ the tumor and tumor-infiltrating immune cell group PD-L1 expression correlates with a good prognosis. The summary of the regression results is shown in Table 3.

Using the new, more inclusive, scoring method for PDL1 expression, we assessed the characteristics of the patients for each type of PD-L1 microenvironment. As shown in Table 4, we found that the majority of cases had a type III PD-L1 microenvironment, which correlates with tumors with constitutive expression.

\section{Discussion}

In the present study, PD-L1 expression was demonstrated in primary uveal melanoma patients. Evaluation of PD-L1 expression has been the focus of much research as it is a key to evaluate the response to anti-PD-L1 therapy in several
Table 4 Type of tumor microenvironment extracted from Teng et al. [49]

\begin{tabular}{lccll}
\hline $\begin{array}{l}\text { Types of } \\
\text { microenviroment }\end{array}$ & PD-L1 & $\begin{array}{c}\text { TIL } \\
\begin{array}{l}\text { Cases total } \\
(\%), N= \\
67\end{array}\end{array}$ & $\begin{array}{l}\text { Cases metastatic } \\
(\%), N=25\end{array}$ \\
\hline Type I & + & + & $7(10.5)$ & $3(12)$ \\
Type II & - & - & $16(23.9)$ & $8(32)$ \\
Type III & + & - & $29(43.3)$ & $7(28)$ \\
Type IV & - & + & $15(22.4)$ & $7(28)$ \\
\hline $\begin{array}{l}P D-L 1 \text { programmed } \\
\text { lymphocytes }\end{array}$ & cell & death-1/ligand, & $T I L$ & tumor-infiltrating \\
& & & &
\end{tabular}

tumors, including skin melanoma, lung, and breast cancer [33-35].

Prior to the commercialization of the E1L3N clone used herein, a study showed the lack of reproducibility and specificity using commercially available polyclonal and monoclonal antibodies from a first generation of anti-PD-L1 antibodies [20]. The only antibody that showed good reproducibility in that study was the non-commercial clone 5H1. Moreover, contradictory PD-L1 expression results have been observed in the OCM-1 uveal melanoma cell line in different studies using the aforementioned firstgeneration commercial antibodies [18, 19]. A second generation of rabbit monoclonal antibodies has emerged, enhancing reproducibility, including the E1L3N, SP142, SP263, 28-8, and 22C3 clones [36, 37]. Nevertheless, a standard evaluation or test has not been reached yet. We strongly believe that the E1L3N clone of the anti-PD-L1 antibody has good specificity and could be used to correlate and compare results for different studies in UM from around the world.

Our data demonstrate that PD-L1 expression can be measured in uveal melanoma using immunohistochemistry. Previously, there were only five uveal melanoma cases evaluated using the MIH1 clone and none of these cases showed positivity for PD-L1 [18]. As previously stated, the sensitivity and specificity of the MIH1 clone has been questioned [20]. Our results show that $40-55 \%$ of the UM cases, depending on the threshold used, expressed the PDL1 protein. Furthermore, the staining pattern was heterogeneous in most of the cases, a feature that should not be overseen when studying PD-L1 expression as a tumoral prognostic factor in cytologic samples [38, 39]. It will be important to establish a cutoff value that truly predicts the response to PD-L1 checkpoint inhibitors in future clinical trials with tumoral PD-L1 evaluation.

The importance of the positivity in uveal melanoma cases does not solely rely on the prognosis value of the marker. In vitro studies have shown that uveal melanoma cell-related PD-L1 negatively influences T cell activation, even under interferon- $\boldsymbol{\gamma}$-free (control) conditions [40], 
producing an immune evasive state. Moreover, activation of the PD-1/PD-L1 pathway in uveal melanoma inhibits T cell activation in vivo [41].

As PD-L1 expression in primary uveal melanoma was not previously achieved by immunohistochemistry, we correlate our results to the mRNA expression of PD-L1 using the SurvExpress bioinformatic tool [42]. Two cohorts of uveal melanoma with PD-L1 mRNA expression were found: one of them showed a trend of good prognosis with PD-L1 expression ( $n=41, P=0.2$, Worley, Hammond, LA, USA, uveal melanoma GSE39717, Supplementary image 1). These results further support investigation of PDL1 as a prognostic marker in a large cohort of uveal melanoma. On the other hand, the second cohort did not show any difference between PD-L1 expression and overall survival ( $P=0.7$, Laurent Saule Uveal Melanoma GSE22138).

Contrary to previous studies where PD-L1 expression was associated with a poor prognosis in solid tumors [43, 44], our data indicated that PD-L1 is associated with a good prognosis. Moreover, we observed that PD-L1 expression in uveal melanoma is associated with a decrease of tumorinfiltrating lymphocytes, also in opposition to other solid tumors in which PD-L1 is associated with an increase of tumor-infiltrating lymphocytes $[45,46]$. To understand this paradox, it is important to consider that the eye is an immune-privileged site.

First, there are no lymphatics in the eye and its capability to control and inhibit immune responses is well known [47]. It has been hypothesized that the presence of lymphocytes in the eye occurs after malignant cells have been presented to the immune system at extraocular sites. This theory was stablished to explain why the presence of lymphocytes is associated with poor prognosis [48], which is contrary to other solid tumors, including skin melanoma.

Second, to interpret PD-L1 expression, it is important to remember that there are two types of expression: constitutive and adaptive. In order to recognize these different types of expression morphologically, Teng et al. [49] described four categories, which are summarized in Table 4. Type I is the most likely to respond to a single checkpoint inhibitor and correlates with an adaptive response. On the other hand, Type II is associated with tumors that do not induce an immune response. Type III relates to tumors with a constitutive expression and type IV are the tumors where other immune suppressive pathways might dominate. The last three types are associated with poor response to a single checkpoint inhibitor. As most of the cases of uveal melanoma had a type III microenvironment, we can hypothesize that in most uveal melanoma cases a constitutive expression is more likely associated to a good prognosis. In aggressive cases, melanoma cells leave the eye and induce an immune response towards the tumor. In these cases, the adaptive tumoral immune response switches from PD-L1 to another checkpoint inhibitor, such as indoleamine-pyrrole 2,3 dioxygenase, CTLA-4, or L-RAS [47].

In order to deeply analyze this hypothesis, we compared our results to a meta-analysis of anti-PD-1 clinical trials in which 56 patients with uveal melanoma metastasis were treated [50]. For none of the cases an evaluation of the PDL1 microenvironment status was performed. In this metaanalysis, only $13 \%$ of the patients presented with at least a stable disease for more than 6 months. Analyzing our series, we found that $12 \%$ of the metastatic tumors had a type I of PD-L1 microenvironment, which is the type associated with a therapeutic response to single checkpoint inhibitor therapy. These results highlight the importance of evaluating PD-L1 expression to select patients that are prone to a positive response, hence the underlying concept of personalized medicine.

Furthermore, it is well known that PD-L1 expression in a tumor can be modified after treatment. It has been suggested that even for tumors with non-favorable PD-L1 expression patterns (types II to IV), patients may benefit from anti-PDL1 therapy when used in combination either with radiotherapy or combined immunotherapy [51-53]. Further studies should evaluate the role of anti-PD-L1 therapy in combined treatment regimens.

A limitation of the present study is the lack of uniformity of follow-ups between patients. However, this variable was accounted for in our statistical analysis and was considered when creating our model of prediction. Moreover, the fixation time was not uniform and some of the paraffin blocks dated from over 10 years ago. To address these limitations, all cases had an internal control that allowed us to assess the antigenicity of each sample. Also, our study group encompasses only patients who underwent enucleation; patients with small tumors might not be well represented in our cohort.

Another limitation is that the evaluation of the nonneoplastic cells was performed using only morphological basis without possibilities to sub-classify the lymphocytes or the macrophages. Further studies using immunofluorescence to evaluate the tumor inflammatory microenvironment will help us understand better the relationship between PD-L1 expression and lymphocytes or macrophages, respectively.

Moreover, it is well known that tumors can change or modify their microenvironment between the primary tumor and the metastasis. It would be interesting to obtain samples from the metastases to assess whether there is a good correlation between primary tumors and metastatic disease.

In summary, the E1L3N clone of the anti-PD-L1 monoclonal antibody appears to be a specific marker for PD-L1 in cases of uveal melanoma. Its expression correlates with a better outcome and decreased tumor-infiltrating lymphocytes. Moreover, it has the potential to identify 
subgroups of uveal melanoma patients that might benefit from PD-1/PD-L1 pathway blockade. The ability to predict potential uveal melanoma responders based on their PD-L1 expression pattern needs to be further evaluated and validated in biomarker-targeted immunotherapy-associated clinical trials, bringing us one step closer to personalized medicine and treatment.

Acknowledgements We thank Ms. Juliana Passos for their support in the ocular pathology database search, and Sonia Corredor MD and William April for their help on the database acquisition, Sabrina Bergeron for her help in reviewing the manuscript, and Ms. Tiffany Porraccio for her support in administrative paperwork. The present work was funded by Henry Shibata Fellowship from Cedars Cancer foundation and MITACS.

Funding This work was funded by Henry Shibata Fellowship from Cedars Cancer foundation and MITACS.

\section{Compliance with ethical standards}

Conflict of interest The authors declare that they have no conflict of interest.

\section{References}

1. Singh AD, Topham A. Incidence of uveal melanoma in the United States: 1973-1997. Ophthalmology. 2003;110:956-61.

2. Hawkins BS. The Collaborative Ocular Melanoma Study (COMS) randomized trial of pre-enucleation radiation of large choroidal melanoma: IV. Ten-year mortality findings and prognostic factors. COMS report number 24. Am J Ophthalmol. 2004;138:936-51.

3. The COMS randomized trial of iodine 125 brachytherapy for choroidal melanoma: V. Twelve-year mortality rates and prognostic factors: COMS report No. 28. Arch Ophthalmol. 2006;124:1684-93.

4. Singh AD, Topham A. Survival rates with uveal melanoma in the United States: 1973-1997. Ophthalmology. 2003;110:962-5.

5. Callejo SA, Antecka E, Blanco PL, Edelstein C, Burnier MN Jr.. Identification of circulating malignant cells and its correlation with prognostic factors and treatment in uveal melanoma. A prospective longitudinal study. Eye (Lond). 2007;21:752-9.

6. Fernandes BF, Antecka E, dos Passos G, Burnier MN Jr. Detection of circulating malignant cells in patients with uveal melanoma. Arq Bras Oftalmol. 2007;70:593-8.

7. Logan PT, Fernandes BF, Di Cesare S, et al. Single-cell tumor dormancy model of uveal melanoma. Clin Exp Metastastasis. 2008;25:509-16.

8. Ott PA, Hodi FS, Robert C. CTLA-4 and PD-1/PD-L1 blockade: new immunotherapeutic modalities with durable clinical benefit in melanoma patients. Clin Cancer Res. 2013;19:5300-9.

9. Nguyen LT, Ohashi PS. Clinical blockade of PD1 and LAG3potential mechanisms of action. Nat Rev Immunol. 2015;15:45-56.

10. Butte MJ, Pena-Cruz V, Kim MJ, Freeman GJ, Sharpe AH. Interaction of human PD-L1 and B7-1. Mol Immunol. 2008;45:3567-72.

11. Ribas A, Tumeh PC. The future of cancer therapy: selecting patients likely to respond to PD1/L1 blockade. Clin Cancer Res. 2014;20:4982-4.

12. Khalil DN, Smith EL, Brentjens RJ, Wolchok JD. The future of cancer treatment: immunomodulation, CARs and combination immunotherapy. Nat Rev Clin Oncol. 2016;13:273-90.
13. Topalian SL, Sznol M, McDermott DF, et al. Survival, durable tumor remission, and long-term safety in patients with advanced melanoma receiving nivolumab. J Clin Oncol. 2014;32:1020-30.

14. Khalil DN, Smith EL, Brentjens RJ, Wolchok JD. The future of cancer treatment: immunomodulation, CARs and combination immunotherapy. Nat Rev Clin Oncol. 2016;13:394.

15. Topalian SL, Hodi FS, Brahmer JR, et al. Safety, activity, and immune correlates of anti-PD-1 antibody in cancer. N Engl J Med. 2012;366:2443-54.

16. Gibney GT, Weiner LM, Atkins MB. Predictive biomarkers for checkpoint inhibitor-based immunotherapy. Lancet Oncol. 2016;17:e542-e51.

17. Meng X, Huang Z, Teng F, Xing L, Yu J. Predictive biomarkers in PD-1/PD-L1 checkpoint blockade immunotherapy. Cancer Treat Rev. 2015;41:868-76.

18. Yang W, Chen PW, Li H, Alizadeh H, Niederkorn JY. PD-L1: $\mathrm{PD}-1$ interaction contributes to the functional suppression of Tcell responses to human uveal melanoma cells in vitro. Invest Ophthalmol Vis Sci. 2008;49:2518-25.

19. Ma J, Usui Y, Kezuka T, et al. Costimulatory molecule expression on human uveal melanoma cells: functional analysis of CD40 and B7-H1. Exp Eye Res. 2012;96:98-106.

20. Velcheti V, Schalper KA, Carvajal DE, et al. Programmed death ligand-1 expression in non-small cell lung cancer. Lab Invest. 2014;94:107-16.

21. Ilie M, Hofman V, Dietel M, Soria JC, Hofman P. Assessment of the PD-L1 status by immunohistochemistry: challenges and perspectives for therapeutic strategies in lung cancer patients. Virchows Arch. 2016;468:511-25.

22. Schats KA, Van Vre EA, De Schepper S, et al. Validated programmed cell death ligand 1 immunohistochemistry assays (E1L3N and SP142) reveal similar immune cell staining patterns in melanoma when using the same sensitive detection system. Histopathology. 2017;70:253-63.

23. McLean IW, Foster WD, Zimmerman LE, Gamel JW. Modifications of Callender's classification of uveal melanoma at the Armed Forces Institute of Pathology. Am J Ophthalmol. 1983;96:502-9.

24. Angi M, Damato B, Kalirai $\mathrm{H}$, et al. Immunohistochemical assessment of mitotic count in uveal melanoma. Acta Ophthalmol. 2011;89:e155-60.

25. McLean IW, Burnier MN, Zimmerman L, Jacobiec F, editors. Tumors of the uveal tract. Washington, DC: American Forces Institute of Pathology; 1994. 41-91pp.

26. de la Cruz PO Jr., Specht CS, McLean IW. Lymphocytic infiltration in uveal malignant melanoma. Cancer. 1990;65:112-5.

27. Makitie T, Summanen P, Tarkkanen A, Kivela T. Tumorinfiltrating macrophages $(\mathrm{CD} 68(+)$ cells $)$ and prognosis in malignant uveal melanoma. Invest Ophthalmol Vis Sci. 2001;42:1414-21.

28. Yang W, Li H, Chen PW, et al. PD-L1 expression on human ocular cells and its possible role in regulating immune-mediated ocular inflammation. Invest Ophthalmol Vis Sci. 2009;50:273-80.

29. Taube JM, Klein A, Brahmer JR, et al. Association of PD-1, PD-1 ligands, and other features of the tumor immune microenvironment with response to anti-PD-1 therapy. Clin Cancer Res. 2014;20:5064-74.

30. Santarpia M, Karachaliou N. Tumor immune microenvironment characterization and response to anti-PD-1 therapy. Cancer Biol Med. 2015;12:74-8.

31. Fehrenbacher L, Spira A, Ballinger M, et al. Atezolizumab versus docetaxel for patients with previously treated non-small-cell lung cancer (POPLAR): a multicentre, open-label, phase 2 randomised controlled trial. Lancet. 2016;387:1837-46.

32. Driver BR, Miller RA, Miller T, et al. Programmed death ligand-1 (PD-L1) expression in either tumor cells or tumor-infiltrating 
immune cells correlates with solid and high-grade lung adenocarcinomas. Arch Pathol Lab Med. 2017;141:1529-32.

33. Sun WY, Lee YK, Koo JS. Expression of PD-L1 in triple-negative breast cancer based on different immunohistochemical antibodies. J Transl Med. 2016;14:173.

34. Wang X, Teng F, Kong L, Yu J. PD-L1 expression in human cancers and its association with clinical outcomes. Onco Targets Ther. 2016;9:5023-39.

35. Kerr KM, Nicolson MC. Non-small cell lung cancer, PD-L1, and the pathologist. Arch Pathol Lab Med. 2016;140:249-54.

36. Sholl LM, Aisner DL, Allen TC, et al. Programmed death ligand-1 immunohistochemistry - a new challenge for pathologists: a perspective from Members of the Pulmonary Pathology Society. Arch Pathol Lab Med. 2016;140:341-4.

37. Hirsch FR, McElhinny A, Stanforth D, et al. PD-L1 immunohistochemistry assays for lung cancer: results from phase 1 of the Blueprint PD-L1 IHC Assay Comparison Project. J Thorac Oncol. 2017;12:208-22.

38. Madore J, Vilain RE, Menzies AM, et al. PD-L1 expression in melanoma shows marked heterogeneity within and between patients: implications for anti-PD-1/PD-L1 clinical trials. Pigment Cell Melanoma Res. 2014;28:245-53.

39. Correa ZM, Augsburger JJ. Sufficiency of FNAB aspirates of posterior uveal melanoma for cytologic versus GEP classification in 159 patients, and relative prognostic significance of these classifications. Graefes Arch Clin Exp Ophthalmol. 2014;252:131-5.

40. Jia R, Jiao Z, Xu X, et al. Functional significance of B7-H1 expressed by human uveal melanoma cells. Mol Med Rep. 2011;4:163-7.

41. Dong H, Strome SE, Salomao DR, et al. Tumor-associated B7-H1 promotes T-cell apoptosis: a potential mechanism of immune evasion. Nat Med. 2002;8:793-800.

42. Aguirre-Gamboa R, Gomez-Rueda H, Martinez-Ledesma E, et al. SurvExpress: an online biomarker validation tool and database for cancer gene expression data using survival analysis. PLoS ONE. 2013;8:e74250

43. Wang Q, Liu F, Liu L. Prognostic significance of PD-L1 in solid tumor: an updated meta-analysis. Medicine (Baltim). 2017;96: e6369.

44. Guan J, Lim KS, Mekhail T, Chang CC. Programmed death ligand-1 (PD-L1) expression in the programmed death receptor-1 (PD-1)/PD-L1 blockade: a key player against various cancers. Arch Pathol Lab Med. 2017;141:851-61.

45. Ameratunga M, Asadi K, Lin X, et al. PD-L1 and tumor infiltrating lymphocytes as prognostic markers in resected NSCLC. PLoS ONE. 2016;11:e153954.

46. Wimberly H, Brown JR, Schalper K, et al. PD-L1 expression correlates with tumor-infiltrating lymphocytes and response to neoadjuvant chemotherapy in breast cancer. Cancer Immunol Res. 2015;3:326-32.

47. Niederkorn JY. Immune escape mechanisms of intraocular tumors. Prog Retin Eye Res. 2009;28:329-47.

48. Whelchel JC, Farah SE, McLean IW, Burnier MN. Immunohistochemistry of infiltrating lymphocytes in uveal malignant melanoma. Invest Ophthalmol Vis Sci. 1993;34:2603-6.

49. Teng MW, Ngiow SF, Ribas A, Smyth MJ. Classifying cancers based on T-cell infiltration and PD-L1. Cancer Res. 2015;75:2139-45.

50. Algazi AP, Tsai KK, Shoushtari AN, et al. Clinical outcomes in metastatic uveal melanoma treated with PD-1 and PD-L1 antibodies. Cancer. 2016;122:3344-53.

51. Kang J, Demaria S, Formenti S. Current clinical trials testing the combination of immunotherapy with radiotherapy. J Immunother Cancer. 2016;4:51.

52. Bu X, Mahoney KM, Freeman GJ. Learning from PD-1 resistance: new combination strategies. Trends Mol Med. 2016;22:448-51.

53. Larkin J, Chiarion-Sileni V, Gonzalez R, et al. Combined nivolumab and ipilimumab or monotherapy in untreated melanoma. $\mathrm{N}$ Engl J Med. 2015;373:23-34. 